\title{
How are countries in sub-Saharan African monitoring the impact of programmes to prevent vertical transmission of HIV?
}

\author{
Ameena Goga and colleagues describe how five countries in sub-Saharan Africa are monitoring \\ the effectiveness of national programmes to prevent vertical transmission of HIV
}

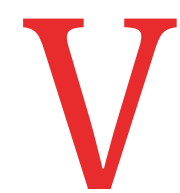
ertical transmission of HIV can occur during pregnancy, delivery, or through breast feeding. The main driver of vertical transmission is a high maternal viral load. ${ }^{1}$ Between 2002 and 2016, low and middle income countries (LMICs) in sub-Saharan Africa with high HIV prevalence improved their policies to prevent vertical transmission of HIV. In 2002, national policies recommended single dose nevirapine at the onset of labour, with limited or no breast feeding. By 2016, all Global Plan priority countries in sub-Saharan Africa (where $90 \%$ of the world's HIV positive pregnant women live) had adopted Option B+ with promotion of breast feeding. ${ }^{2}$ Option $\mathrm{B}+$ was a dramatic policy change recommending lifelong triple antiretroviral therapy (ART) for all pregnant and lactating women living with HIV. The aim is to protect the child from

\section{KEY MESSAGES}

- Monitoring vertical transmission of HIV is important in high HIV prevalence low and middle income countries where health systems are often weak or stretched beyond capacity

- WHO recommends three methods to measure the effectiveness of programmes that prevent vertical transmission of HIV: statistical modelling, surveys or surveillance, and analysis of programme data

- Kenya, Malawi, South Africa, Uganda, and Zimbabwe all use statistical modelling to monitor programme effectiveness

- All countries are strengthening routine programmatic monitoring, which requires additional systems such as allocating unique identifiers, and developing interclinic data linkages to trace clients who transfer between facilities
HIV infection, ensure the mother's future health, and prevent horizontal transmission of HIV.

Monitoring country level effectiveness of these policy changes is critical, both to ensure they are making a difference to people's lives and to track progress towards achieving global goals. These goals include the Sustainable Development Goal of ending the AIDS epidemic by 2030; the Global Plan goals of reducing new childhood HIV infections by $90 \%$ and HIV related child deaths by more than $50 \%$; and the goal of eliminating transmission of HIV between a mother and her child so that the transmission rate at the end of breast feeding is less than $5 \%$ and new paediatric HIV infections are $\leq 50$ per 100000 live births. ${ }^{23}$ Such monitoring is particularly important in LMICs where HIV prevalence is high and where health systems are often weak or stretched beyond capacity.

The World Health Organization recommends three methods to measure programme effectiveness: statistical modelling, surveys or surveillance, and analysis of routine programme data (table 1). ${ }^{4}$ This paper looks at how five countries with high HIV prevalence, prioritised for elimination of vertical transmission, have translated global guidance on monitoring prevention programme effectiveness into action.

Our analysis is based on document reviews, ${ }^{23}$ peer reviewed publications, reports, and a consultation convened by the South African Medical Research Council in 2016. Representatives from five countries (Kenya, Malawi, South Africa, Uganda, and Zimbabwe) attended the consultation, alongside colleagues from key multilateral agencies including the Joint United Nations Programme on HIV and AIDS (UNAIDS), WHO, the Interagency Task Team on the Prevention and Treatment of HIV Infection in Pregnant Women, Mothers, and their Children, and Unicef.

\section{Country approaches and lessons learnt \\ Statistical modelling}

Models are mathematical equations that can be used to predict outcomes that are difficult to measure directly, such as national HIV incidence and vertical transmission of HIV. Spectrum is a suite of user friendly, easily implementable, regularly updated, free models that support decision making by policy makers (www.avenirhealth.org/ software-spectrum.php). Spectrum consists of several software models including the Lives Saved Tool, which focuses on child survival, and the Aids Impact Model (AIM). Other HIV related models, outside of the Spectrum suite, include the Asian Epidemic model, the Thembisa model (which models the South Africa epidemic), ${ }^{5}$ and the Global Burden of Disease (GBD) estimates, drawn from vital registration systems. Until now, however, GBD data have not been used to track effectiveness of prevention of vertical transmission. ${ }^{6}$ Only Spectrum is available for use by country teams, who are trained regularly, allowing country level tracking and intercountry comparison. The Spectrum AIM uses HIV sentinel and population based surveillance data and programme data in demographic models to calculate estimates. It can estimate vertical transmission of HIV, the number of children living with HIV, the number of new HIV infections in children, and HIV related mortality. Estimates, however, are only as valid as the data and assumptions that go into the models. Input data are usually drawn from prevalence in high risk groups and an estimation of the proportion of the population in these groups. ${ }^{6}$ Spectrum software is used by national programmes and UNAIDS to prepare estimates for 161 countries, including Kenya, Malawi, South Africa, Uganda, and Zimbabwe.

In these countries the Spectrum model is used to estimate early (six weeks post-delivery) and long term (six weeks to 24 months) effectiveness of vertical transmission prevention programmes. In fact, because no routine cohort monitoring 
systems exist in LMICs with high HIV prevalence, modelling has been the only method consistently used to estimate long term prevention of vertical transmission effectiveness. Until routine data sources are more reliable and of better quality, modelling is needed to estimate the impact of HIV programmes. Modelling is also needed to forecast HIV disease burden to inform future health needs and service provision.

\section{Surveys or surveillance}

Guidance from WHO recommends intermittent immunisation clinic surveys, household surveys, or surveillance through demographic surveillance sites to measure the impact of vertical transmission programmes in prevention. ${ }^{4}$ Immunisation clinic surveys can be conducted at national or subnational level to measure vertical transmission of HIV (up to six weeks postdelivery), number of children living with or exposed to HIV, and vertical transmis- sion of HIV at the end of breast feeding, if children can be followed up at later time points. Immunisation clinic surveys can provide cross sectional and trend data in settings with high immunisation coverage and allow the collection of coverage and outcome data among all children, not only children born to mothers who are known to have HIV and have interacted with prevention programmes. ${ }^{489}$ Immunisation surveys can be expensive, however, especially if more staff need to be hired, and they can underestimate vertical transmission of HIV as recruitment usually occurs after the neonatal period, thus excluding early infections and deaths.

Malawi, South Africa, and Zimbabwe have conducted cross sectional surveys at immunisation clinics to measure prevention of vertical transmission effectiveness (table 2), but only Zimbabwe and South Africa have generated nationally representative estimates. These surveys are designed to enrol a clinic attending population of mother-infant pairs, regardless of HIV status and interaction with prevention services (table 1). This mitigates selection bias, enrolling mothers of unknown HIV status and recently diagnosed mothers. Measurements have included infant HIV exposure, presumed or actual maternal HIV positivity, vertical transmission of HIV, uptake of prevention interventions, adherence to maternal ART, and uptake of routine maternal and child healthcare (table 1).

When designing clinic based surveys, researchers need to plan for the return of the mother's and infant's HIV test results and need to link participants to appropriate care, as it is unethical for these surveys to be anonymised or unlinked.

In Malawi, during 2011, a survey was conducted in immunisation clinics within four of the 28 districts; these four districts were purposively selected to reflect regional diversity. ${ }^{10}$ Of the 5068 samples included in the analysis from infants less than 3

\begin{tabular}{|c|c|c|}
\hline Method & Strengths & Limitations \\
\hline $\begin{array}{l}\text { Statistical modelling (Spectrum } \\
\text { model, for example) }\end{array}$ & $\begin{array}{l}\text { Easy to implement } \\
\text { Estimates for previous years can be updated } \\
\text { Provides data for country reports } \\
\text { Use of a similar methodology across years enables inter-year comparison } \\
\text { and tracking of progress } \\
\text { vertical transmission, child survival by time of infection (perinatally, under } \\
6 \text { months, } 7-12 \text { months, and } 12+\text { months after birth), differential vertical } \\
\text { transmission of HIV by maternal CD } 4 \text { count, and the effects of incident infec- } \\
\text { tions and new prophylaxis options }\end{array}$ & $\begin{array}{l}\text { Requires reliable input data. Estimates are, therefore, } \\
\text { only as valid and up to date as the data they use }\end{array}$ \\
\hline \multicolumn{3}{|l|}{ Surveys or surveillance } \\
\hline $\begin{array}{l}\text { Cross sectional immunisation } \\
\text { clinic survey follow-up }\end{array}$ & $\begin{array}{l}\text { Early immunisation uptake } \\
\text { Can capture known and unknown HIV exposed infants and HIV unexposed } \\
\text { infants } \\
\text { Can link mothers and babies to HIV related care } \\
\text { Can be set up for national, provincial, and district level estimates }\end{array}$ & $\begin{array}{l}\text { Survival bias-no capture of mothers or babies that } \\
\text { died } \\
\text { Selection bias-does not capture mothers who do } \\
\text { not seek facility based care } \\
\text { Can be expensive to implement if data collectors } \\
\text { need to be hired and transported to facilities }\end{array}$ \\
\hline $\begin{array}{l}\text { Health and demographic senti- } \\
\text { nel surveillance }\end{array}$ & $\begin{array}{l}\text { Allows linking of mother-baby pairs and measurement of long term } \\
\text { outcome } \\
\text { Can obtain final vertical transmission of HIV outcomes and relate it to ART } \\
\text { and feeding, and to population HIV estimates }\end{array}$ & $\begin{array}{l}\text { Resource intensive (time, human resources, and cost) } \\
\text { Depending on site selection methods, may not } \\
\text { always be nationally representative }\end{array}$ \\
\hline $\begin{array}{l}\text { Population based impact } \\
\text { surveys }\end{array}$ & $\begin{array}{l}\text { Generalisable to whole population } \\
\text { Collect other measures of programme outputs and behaviours } \\
\text { Already exist in the form of demographic and health surveys or multiple } \\
\text { indicator cluster surveys }\end{array}$ & $\begin{array}{l}\text { Needs large sample size } \\
\text { Requires HIV status of mother and infant } \\
\text { Expensive to implement } \\
\text { Existing surveys have multiple modules and } \\
\text { outcomes and there are few resources for additional } \\
\text { questions and blood tests for mother-infant pairs }\end{array}$ \\
\hline \multicolumn{3}{|c|}{ Use of routine programmatic data } \\
\hline $\begin{array}{l}\text { Routine early infant diagnosis } \\
\text { (EID) and child HIV testing data } \\
\text { from laboratory }\end{array}$ & - EID laboratory databases usually exist & $\begin{array}{l}\text { Needs to be coupled with estimated number or per- } \\
\text { centage of children with no HIV test and their outcomes, } \\
\text { to obtain a national estimate } \\
\text { EID data are not always nationally representative and } \\
\text { exclude HIV positive mothers who do not know they are } \\
\text { positive } \\
\text { Biased from health seeking behaviour } \\
\text { Survival bias, as cannot include children who died } \\
\text { EID does not tackle final transmission, and cannot } \\
\text { link mother to child without unique identifiers }\end{array}$ \\
\hline Cohort follow-up & $\begin{array}{l}\text { Allows linking of mother-baby pairs and measurement of long term } \\
\text { outcome } \\
\text { and feeding }\end{array}$ & $\begin{array}{l}\text { Attrition to follow-up necessitates assumptions dur- } \\
\text { ing analysis } \\
\text { Difficult to trace without unique identifiers } \\
\text { Needs functional longitudinal registers } \\
\text { Resource intensive (time, human resources, and cost) }\end{array}$ \\
\hline
\end{tabular}




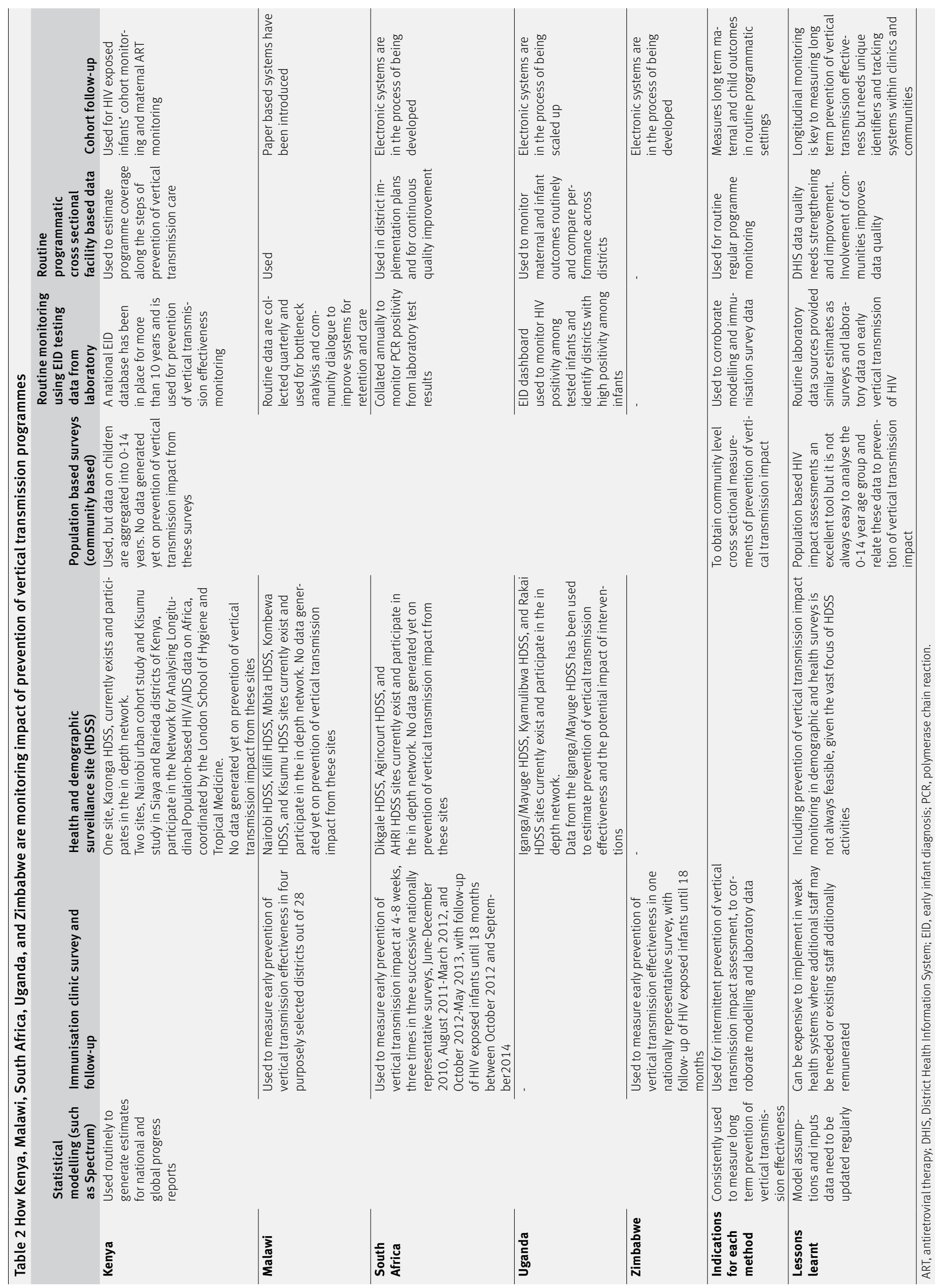


months old, 15.1\% (95\% confidence interval, 14.1 to 16.1 ) were HIV antibody positive. Vertical transmission of HIV was 8.5\% (95\% CI, 6.6 to 10.7) with significant district level differences.

In South Africa, three nationally and provincially representative surveys were conducted at immunisation service points between 2010 and 2013, during the implementation of two different policies to prevent vertical transmission of HIV. ${ }^{89}$ The surveys demonstrated a reduction in vertical transmission of HIV from an estimated $>25 \%$ in the absence of vertical transmission prevention interventions to $2.6 \%$ in 2012 13; vertical transmission of HIV risk differed by prevention of vertical transmission regimen. ${ }^{11}$ The South Africa surveys were expensive to implement; survey data collectors were hired because health facility staff were overburdened and could not incorporate these activities into their daily work. In 2012-2014 longitudinal follow-up of HIV exposed infants was conducted until 18 months to assess long term prevention of vertical transmission effectiveness. Although South Africa implements the regular South Africa Demographic and Health Survey, by 2016 the survey aims were already wide and questionnaires long; thus, monitoring the effectiveness of vertical transmission prevention using this survey has not been feasible.

In Zimbabwe, a nationally representative survey to measure effectiveness of vertical transmission prevention was conducted with 18 month follow-up of mother-infant pairs from facility to community. Verbal autopsies were conducted to understand infant deaths. Cumulative vertical transmission of HIV risk by 18 months was $7 \%$, and data on the differential vertical transmission risk by maternal prevention regimen and infant birth weight have been published..$^{12}$ Unlike in South Africa, the Zimbabwean survey was implemented by health facility staff who received a small allowance for their efforts, making the Zimbabwean survey less expensive.

Household surveys and population based HIV impact assessments provide community level data on the HIV epidemic, obviating the inherent selection bias in facility based surveys (table 2). They have great potential for periodically monitoring the effectiveness of vertical transmission prevention programmes every three to five years in settings where adult HIV prevalence is greater than $2 \%$. The Ugandan, Malawian, and Zimbabwean impact assessments included 10652, 9952, and 9627 children aged 0-14, respectively, but analyses have not yet focused on the 0-14 year group, and have not yet been used to estimate vertical transmission of HIV. ${ }^{13-15}$ The wide paediatric age range in current survey designs restricts their reliability and validity for monitoring prevention programme impact.

Monitoring prevention of vertical transmission through longitudinal surveillance at demographic surveillance sites is theoretically possible. Setting sites up is expensive, however, making the use of existing sites for vertical transmission prevention monitoring easier and less expensive. Although demographic surveillance sites exist in four of the five countries (table 2), as far as we are aware these data have only been used to measure effectiveness of prevention of vertical transmission in Uganda. ${ }^{16}$ Using data from 68000 people from 12000 households, Larsson and colleagues modelled the risk of vertical transmission of HIV among 771 pregnant women in Uganda. ${ }^{16}$ They also modelled the effect of improved prevention coverage on vertical transmission of HIV, demonstrating that $100 \%$ HIV testing coverage or $100 \%$ ART coverage reduces vertical transmission of HIV at birth from $13.4 \%$ to $9.6 \%$ and $11 \%$ respectively. Full (100\%) coverage of antenatal clinic attendance and HIV testing and ART reduces vertical transmission of HIV to $5.4 \%$ at birth. ${ }^{16}$

\section{Using programme data to monitor prevention effectiveness}

WHO recommends three sources of routine data to monitor the impact of vertical transmission prevention programmes, namely routine early infant diagnosis laboratory data; retrospective or prospective cohort data; and case reporting. ${ }^{4}$ However, most high HIV prevalence settings in LMICs lack systems for routine cohort monitoring and rely on routine cross sectional data to monitor prevention effectiveness.

\section{Cross sectional routine data}

Routine programme data are usually available at facility, regional, and national levels (table 2), and they are used in Kenya, Malawi, Uganda, and South Africa to assess pregnant women's access to HIV testing, HIV positive pregnant women's access to ART, HIV exposed infants' access to HIV testing, and infant HIV positivity (table 2). In Malawi, data are also collected on ART initiation and retention at 12, 24, and 36 months post-delivery. among pregnant women with HIV, and vertical transmission of HIV at 6 weeks, 12 months, and 24 months postdelivery. The reports are collected quarterly and used to identify missed opportunities, implement continuous quality improvement, and enhance retention in care.

A case study in Malawi demonstrated that involving community leaders, health surveillance assistants, and healthcare personnel in data review increased programme effectiveness. ${ }^{17}$ In South Africa, District Health Information System (DHIS) and National Health Laboratory Services data are reviewed regularly and reported on at least annually. ${ }^{1819}$ DHIS data demonstrate significant increases in access to maternal ART with concomitant decreases in early polymerase chain reaction (PCR) positivity at six weeks post-delivery over the past five years. ${ }^{19}$ Although routine data are readily available, data accuracy is low. ${ }^{20}$ South Africa, Malawi, Uganda, and Kenya also use laboratory data to document trends in PCR positivity. ${ }^{18}$ Recent analyses in South Africa demonstrate comparability between laboratory generated and survey generated vertical transmission of HIV estimates. ${ }^{21}$ Laboratory data cannot, however, provide information on drivers of vertical transmission as laboratory forms do not capture maternal risk factors, exposure to prevention interventions, or infant feeding patterns, and rely on clinicians to complete information such as infant age. Furthermore, laboratory data need to be de-duplicated, as they often contain repeat infant HIV tests. ${ }^{22}$

\section{Cohort monitoring}

Routine cohort monitoring facilitates the tracking of long term (six weeks to 24 months) prevention of vertical transmission outcomes (table 1). Kenya, Malawi, and Uganda have piloted cohort monitoring of mothers on ART and HIV exposed infants from birth (table 2). Such monitoring enhances maternal retention at 3, 6 , and 12 months, as missed opportunities are recognised easily and corrective action can be taken at clinic and community levels. In Uganda, the HIV Exposed Infants Birth Cohort Analysis monitors HIV exposed infants' service uptake at critical time points ( 6 weeks, 9 and 18 months) and outcomes at 9 and 18 months. Over three years of implementation of the analysis, the proportion of HIV exposed infants retained in care at 18 months increased from $83 \%$ in 2012 to $93 \%$ in 2014 (personal communication, Linda Nabitaka, Ministry of Health, Kampala, Uganda).

\section{Conclusions}

Countries have mainly used modelling with Spectrum software, immunisation clinic 
surveys, and routine cross sectional data to monitor vertical transmission of HIV prevention programme effectiveness. With recent expansion to routine cohort monitoring in three countries, however, several additional opportunities exist to monitor programmes. These include population based HIV impact assessments, health and demographic sentinel surveillance, demographic and health surveys, and laboratory data. There is a critical need to strengthen routine cohort monitoring as this is the most sustainable approach to measure long term effectiveness of vertical transmission prevention programmes.

Contributors and sources: AG conceptualised the paper and led the writing. SW provided information about Zimbabwe. RW provided information about Kenya. ME and WN provided information about Malawi. LN provided information about Uganda. WC, YS, and YP provided information about South Africa. All authors read the paper, contributed to all drafts, and approved the final version of the paper.

Competing interests: We have read and understood BMJ policy on declaration of interests and have no relevant interests to declare. This paper was funded by the South African Medical Research Council (SAMRC). The views expressed in this paper are not the views of either organisation, but of the authors.

Provenance and peer review: Commissioned; externally peer reviewed.

These articles are part of a series proposed by the South African Medical Research Council and commissioned by The BMJ, which retained full editorial control over external peer review, editing, and publication. Open access fees are funded by SAMRC.

Ameena Goga, chief specialist scientist deputy director ${ }^{1,2}$

Yagespari Singh, senior scientist ${ }^{1}$

Debra Jackson, technical adviser ${ }^{3,4}$

Solomon Mukungunugwa, deputy national coordinator $^{5}$

Rose Wafula, PMTCT programme manager ${ }^{6}$

Michael Eliya, PMTCT coordinator ${ }^{7}$

Wingston Felix Ng'ambi, lecturer in epidemiology ${ }^{8}$

Linda Nabitaka, medical officer ${ }^{9}$

Witness Chirinda, specialist scientist ${ }^{1}$

Sanjana Bhardwaj, technical adviser ${ }^{10}$

Shaffiq Essajee, senior adviser ${ }^{11}$

Chika Hayashi, technical adviser ${ }^{11}$

Yogan Pillay, deputy director genera ${ }^{12}$

${ }^{1}$ Health Systems Research Unit, South African

Medical Research Council, South Africa

${ }^{2}$ Department of Paediatrics, University of Pretoria, South Africa

${ }^{3}$ School of Public Health, University of the Western Cape, Cape Town, South Africa

${ }^{4}$ Unicef, Health Section, New York, USA

${ }^{5}$ PMTCT, Paediatric HIV Care Treatment, AIDS and TB Unit, Ministry of Health, Harare, Zimbabwe

${ }^{6}$ National Aids and STI Control Program, Kenya

${ }^{7}$ Ministry of Health, HIV and AIDS Department,

Lilongwe, Malawi
${ }^{8}$ Health Economics and Policy Unit, Department of Health Systems and Policy, College of Medicine, Lilongwe Campus, University of Malawi, Lilongwe, Malawi

${ }^{9}$ Ministry of Health, Kampala, Uganda

${ }^{10}$ Unicef Nigeria, formerly Unicef Pretoria, South Africa

${ }^{11}$ Data and Analytics Section, Unicef, New York, USA

${ }^{12}$ HIV/AIDS, TB, MCWHN, National Department of Health, Pretoria, South Africa

Correspondence to: A Goga

Ameena.Goga@mrc.ac.za

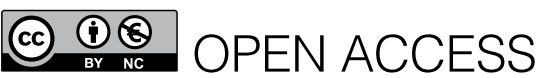

This is an Open Access article distributed in accordance with the Creative Commons Attribution Non Commercial (CC BY-NC 4.0) license, which permits others to distribute, remix, adapt, build upon this work non-commercially, and license their derivative works on different terms, provided the original work is properly cited and the use is non-commercial. See: http://creativecommons.org/ licenses/by-nc/4.0/.

\section{Check for updates}

1 World Health Organization. Use of antiretroviral drugs for treating pregnant women and preventing HIV infection in infants. 2012. www.who.int/hiv/pub/ $\mathrm{mtct} /$ programmatic update2012/en.

2 UNAIDS. On the fast track to an AIDS free generation: the incredible journey of the global plan towards the elimination of new HIV infections among children by 2015 and keeping their mothers alive. 2016. www. aidsdatahub.org/fast-track-aids-free-generationunaids-2016.

3 World Health Organization. Global guidance on criteria and processes for validation: elimination of mother-to-child transmission of HIV and syphilis. 2014. www.who.int/hiv/pub/emtct-validationguidance/en.

4 World Health Organization. A short guide on methods: measuring the impact of national PMTCT programmes. 2012. www.who.int/hiv/pub/mtct/ national_pmtct_guide/en.

5 Johnson L, Dorrington R. Thembisa version 4.1: a model for evaluating the impact of HIV/AIDS in South Africa. 2018. www.thembisa.org/content/filedl/ Thembisa4_1report.

6 GBD 2015 HIV Collaborators. Estimates of global, regional, and national incidence, prevalence, and mortality of HIV, 1980-2015: the Global Burden of Disease Study 2015. Lancet HIV 2016;3:e361-87. doi:10.1016/S2352-3018(16)30087-X

7 Stover J, Brown T, Puckett R, Peerapatanapokin W. Updates to the Spectrum/Estimations and Projections Package model for estimating trends and current values for key HIV indicators. AIDS 2017;31(Suppl 1):S5-11. doi:10.1097/ QAD.0000000000001322

8 Goga AE, Dinh TH, Jackson DJ, et al, South Africa PMTCT Evaluation (SAPMCTE) Team. Populationlevel effectiveness of PMTCT Option A on early mother-to-child (MTCT) transmission of HIV in South Africa: implications for eliminating MTCT. J Glob Health 2016;6:020405. doi:10.7189/ jogh.06.020405

9 Goga AE, Dinh TH, Jackson DJ, et al, South Africa PMTCT Evaluation Team. First population-level effectiveness evaluation of a national programme to prevent HIV transmission from mother to child, South Africa. J Epidemiol Community Health 2015;69:2408. doi:10.1136/jech-2014-204535
10 Sinunu MA, Schouten EJ, Wadonda-Kabondo $\mathrm{N}$, et al. Evaluating the impact of prevention of mother-to-child transmission of HIV in Malawi through immunization clinic-based surveillance. PLoS One 2014;9:e100741. doi:10.1371/journal. pone.0100741

11 Goga A, Sherman G, Chirinda W, et al. Eliminating mother-to-child transmission of HIV in South Africa, 2002-2016: progress, challenges, and the Last Mile Plan. South African Health Reviews 2017. www. hst.org.za/publications/South\%20African\%20 Health\%20Reviews/13 Eliminating\%20mother\%20 to $\% 20$ child\%20transmission $\% 20$ of $\% 20$ HIV\%20 in\%20South\%20Africa_2002\%20to\%202016_ progress_challanges $\% 20$ and $\% 20$ the $\% 2$ Last $\% 20$ Mile\%20Plan.pdf.

12 Dinh TH, Mushavi A, Shiraishi RW, et al. Impact of timing of antiretroviral treatment and birth weight on mother-to-child human immunodeficiency virus transmission: findings from an 18-month prospective cohort of a nationally representative sample of mother-infant pairs during the transition from option A to option B+ in Zimbabwe. Clin Infect Dis 2018;66:576-85. doi:10.1093/cid/cix820

13 Ugandan Government. Uganda population based HIV impact assessment. 2017. http://library.health. go.ug/publications/service-delivery-diseasescontrol-prevention-communicable-diseases/hivaids/ uganda- 0 .

14 Malawi Ministry of Health. Malawi population-based HIV impact assessment. 2016. http://phia.icap. columbia.edu/wp-content/uploads/2016/09/ MALAWI-Factsheet.FIN_.pdf.

15 Zimbabwe Ministry of Health and Child Care. Zimbabwe population-based HIV impact assessment. 2016. http://phia.icap.columbia. edu/wp-content/uploads/2016/11/ZIMBABWEFactsheet.FIN_.pdf.

16 Larsson EC, Ekström AM, Pariyo G, et al. Prevention of mother-to-child transmission of HIV in rural Uganda: modelling effectiveness and impact of scaling-up PMTCT services. Glob Health Action 2015;8:26308. doi:10.3402/gha.v8.26308

17 UNICEF. Together we can improve: a case study on joint community-facility review of PMTCT dashboards in Malawi. 2016. www.pedaids.org/resource/a-casestudy-on-joint-community-facility-review-of-pmtctdashboards-in-malawi.

18 Sherman G, Lilian R, Bhardwaj S, Candy S, Barron P. Laboratory information system data demonstrate successful implementation of the prevention of mother-to-child transmission programme in South Africa. S Afr Med I 2014;104(Suppl 1):235-8. doi:10.7196/SAMJ.7598

19 Massyn N, Peer N, English R. District Health Barometer. 2016. www.hst.org.za/sites/default/files/ Complete_DHB_2015_16_linked.pdf.

20 Nicol E, Dudley L, Bradshaw D. Assessing the quality of routine data for the prevention of mother-to-child transmission of HIV: An analytical observational study in two health districts with high HIV prevalence in South Africa. Int J Med Inform 2016;95:60-70. doi:10.1016/j. ijmedinf.2016.09.006

21 Sherman G, Haeri Mazanderani A, Barron P, et al. Toward elimination of mother-to-child transmission of HIV in South Africa: how best to monitor early infant infections within the Prevention of Mother-to-Child Transmission Program. J Glob Health 2017;7:010701. doi:10.7189/ jogh.07.010701

22 Moyo F, Haeri Mazanderani A, Barron P, et al. Introduction of Routine HIV Birth Testing in the South African National Consolidated Guidelines. Pediatr Infect Dis / 2018;37:559-63. doi:10.1097/ INF.0000000000001840

Cite this as: BMJ 2019;364:1660

http://dx.doi.org/10.1136/bmj.1660 